\title{
A Novel Scheme for Spectrum Prediction in Cognitive Radio Networks
}

\author{
Mehdi Askari, Rezvan Dastanian
}

\begin{abstract}
An efficient spectrum prediction model is presented to improve the spectrum utilization in cognitive radio network. In this model, a novel improved version of Teaching-LearningBased-Optimization algorithm, also referred to iTLBO algorithm, is proposed to train a feedforward artificial neural network (ANN). The performance of the proposed iTLBO-ANN model is compared with some hybrid prediction models, including the genetic algorithm with ANN (GA-ANN), the firefly algorithm with ANN (FF-ANN), and the conventional TLBO algorithm with ANN (TLBO- ANN). Performance evaluation via a real-word spectrum dataset (GSM-900) confirms that iTLBO-ANN outperforms other spectrum prediction schemes in terms of prediction error and prediction efficiency.
\end{abstract}

Index Terms - Cognitive radio, Spectrum prediction, Artificial neural network, TLBO, Evolutionary algorithms

\section{INTRODUCTION}

$\mathrm{D}$ UE to the increasing demand for wireless applications such as mobile internet and Internet of Things (IOT) over the past decades, more efficient frequency spectrum management has become a critical challenge. Because of static spectrum regulations, a considerable amount of frequency band remains unused in normal conditions. Therefore, the frequency spectrum efficiency can be significantly reduced. A promising solution for efficient management of frequency spectrum is cognitive radio $(\mathrm{CR})$. In $\mathrm{CR}$, the radio can adjust its parameters according to what becomes aware of, feels and learn about its environment [1-3]. Considering the channel access priority for the authorized or primary users (PUs), a CR user or secondary user (SU) can opportunistically occupy the frequency holes (unused licensed channels) as long as it does not any harmful interference to the PUs.

Spectrum management in a CR network is divided into four major functions including spectrum sensing to understand the spectrum occupancy of PUs, spectrum decision to select the best available channel based on what has been recognized in the spectrum sensing, spectrum sharing to optimally share the spectrum holes with other CR users, and spectrum mobility to

This manuscript is submitted on $6^{\text {th }}$ May 2021 and accepted on $23^{\text {th }}$ August 2021. M. Askari and R. dastanian are with the Electrical Engineering Department, Behbahan Khatam al Anbia University of Technology, Behbahan, Iran (e-mail: askari@bkatu.ac.ir, dastanian@bkatu.ac.ir).

1985-5389/C 2021 The Authors. Published by UiTM Press. This is an open access article under the CC BY-NC-ND license (http://creativecommons.org /licenses/by-nc-nd/4.0/). handoff the spectrum when the current spectrum is required by the returned PU. Since all of these are time and energy consuming functions [4], prediction-based spectrum management have been widely studied in literature [5-10]. The objective of a spectrum prediction technique is to predict the next status of the spectrum based on the current and previous observations. Clearly, having information about the status of frequency channels in different time slots provides valuable insights for regulators and decision makers to make conscious decisions on future spectrum assignment [11]. A CR user can therefore sense those channels that are probably idle, decide and select a high quality channel and leave the channel at the right time based on the predicted behavioral model of users.

In [4], the authors summarize the state of the art of spectrum prediction for the CR networks in an interesting way. However, various techniques have been introduced to predict spectrum occupancy using past spectrum sensing results. A binary time series model is presents in [5] for spectrum occupancy characterization and prediction. In [6], a spectrum prediction model based on the MLP neural network is proposed and its prediction accuracy is compared with a conventional HMM predictor. In [7], the frame structure of SUs are redesigned by adding a spectrum prediction function to select only the channels for sensing that are predicted to be idle. A spectrum prediction scheme for multi-PU multi-SU Cognitive radios is presented in [8]. In this scheme, the cooperative group formation process is modeled as a coalitional game and a coalition formation algorithm is proposed. In [9], the performance of the cooperative spectrum prediction as a function of PUs channel occupancy is investigated using HMM and MLP predictors. In [10], a spectrum entropy prediction method is proposed to select a channel with better stability to avoid frequent interruptions of channel access in CR networks.

In this work, the spectrum data for the GSM-900 downlink band are collected using a radiometer that measures the RSS over the spectrum. The main contributions of this work are summarized as follows:

- An improved Teaching-Learning-Based-Optimization (iTLBO) algorithm is presented.

- A novel hybrid spectrum prediction model is developed. This model uses the proposed iTLBO algorithm to train a multi-layer Artificial-Neural-Network (ANN) weights.

- A real-world spectrum data analysis is presented to evaluate the data correlations in time domain. 
- Several spectrum prediction algorithms, including GAANN, FF-ANN and TLBO-ANN, are also simulated for the real-world data. However, comparing the proposed scheme with other spectrum prediction models confirms the effectiveness and efficiency of the proposed iTLBOANN model.

The rest of the paper is organized as follows. The system model is presented in section 2. Section 3 describes the proposed prediction algorithm. The experimental evaluations are performed in section 4. Finally, Section 5 is a conclusion of this paper.

\section{SYSTEM MODEL}

In a CR network, a radio spectrum segment (a channel) is assigned to each user for data transmit/receive. Since PUs have higher priority to use the licensed spectrum than SUs, the SUs can only use the spectrum channels that are not occupied by PUs. However, if a PU needs a specific channel that has already been occupied by a SU, the SU must immediately leave the channel.

Fig. 1 shows a binary form spectrum data matrix $\mathbf{D} \in \mathbb{R}^{F \times T}$ with $\mathrm{F}$ rows and $\mathrm{T}$ columns. Each row $\mathbf{d}_{i, .}:=\left[d_{i, 1}, d_{i, 2}, \ldots, d_{i, T}\right]$, $d_{i, .} \in\{0,1\}$, and $i \in\{1, \ldots, F\}$, indicates a frequency band and each column $\mathbf{d}_{\mathrm{s}, j}:=\left[d_{1, j}, d_{2, j}, \ldots, d_{F, j}\right]^{\prime}, d_{., j} \in\{0,1\}$, and $j \in$ $\{1, \ldots, T\}$, indicates a time slot, where $(.)^{\prime}$ represents the transpose operation. A time slot $\mathbf{d}_{, j}$ denotes the state distribution of $F$ frequency bands. Each matrix element $d_{i, j}, i \in$ $\{1, \ldots, F\}, j \in\{1, \ldots, T\}$ shows the stat of the $i$ th frequency band in the $j$ th time slot. Taking spectrum occupancy status of $T-1$ previous time slots into account, the objective of spectrum prediction is to forecast the spectrum status of $T$ th time slot [11].

To characterize the spectrum state, a radiometer is used to sense the spectrum and measure the power spectral density (PDS) of frequency bands. The measured spectrum data $x$ at $i$ th frequency band in the $j$ th time slot is denoted as

$$
x_{i, j}=s_{i, j}+w_{i, j}
$$

where $s_{i, j}$ represents the PDS of the signal of interest, and $w_{i, j}$ is additive noise component with zero mean and variance $\sigma^{2}$. In the absence of a signal, only the noise component is measured by radiometer as

$$
x_{i, j}=w_{i, j}
$$

Each measured data is then compared with a detection threshold $k$ to obtain binary spectrum occupancy (BSO) information as follow

$$
d_{i, j}= \begin{cases}1, & x_{i, j}>k \\ 0, & x_{i, j}<k\end{cases}
$$

where $d_{i, j}$ is the spectrum data matrix element in the $i$ th frequency band and the $j$ th time slot as mentioned above. Due

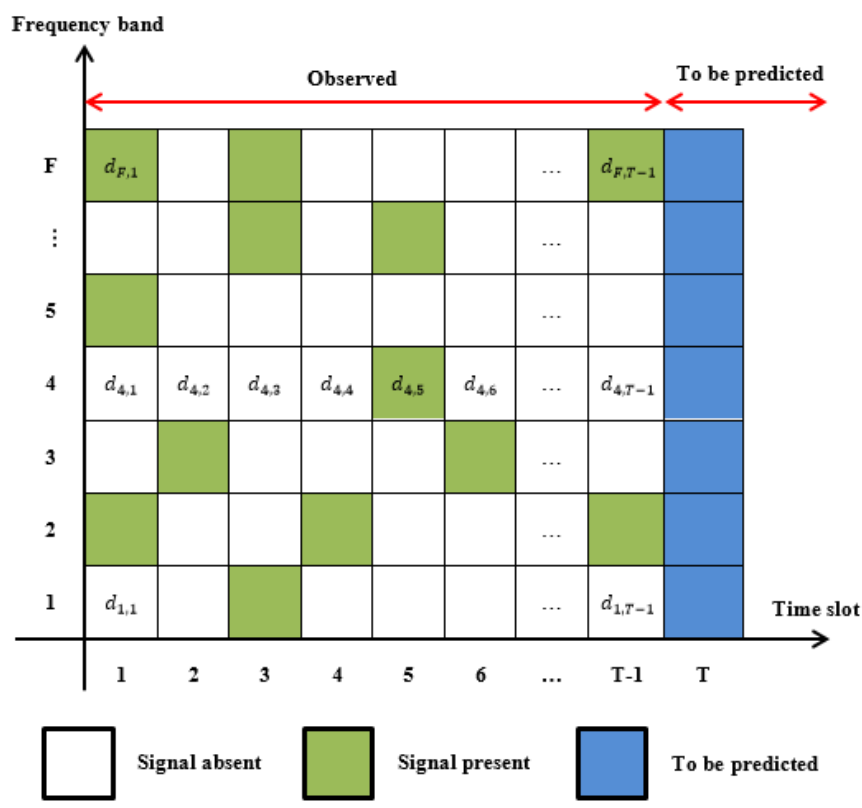

Fig. 1. A binary form spectrum data matrix

to several environmental factors such as fading, shadowing, and so on, the validity of the spectrum occupancy information should be checked. There are some effective methods to provide reliable spectrum sensing in literature [12-14]. Assuming reliable data sensing is provided by one of the reliable methods, the noise floor (NF) is selected as detection threshold $k$ in this study.

\section{PROPOSED ALGORITHM}

Due to the importance of training process in ANN, robust optimization techniques can be used to attain more accurate and efficient performances. Based on the results reported in [15-17], teaching-learning-based optimization (TLBO) is an efficient and accurate optimization method and shows superior performance in comparison with some other naturally inspired optimization algorithms such as genetic algorithm (GA) [18], particle swarm optimization (PSO) [19], differential evolution (DE) [20], artificial bee colony (ABC) [21] and firefly algorithm (FA) [22]. TLBO algorithm has some remarkable advantages such as simple implementation, low computational complexity, high ability to find the global optimum, fewer required control variables, and low dependency of the final solution convergence to the initial population.

In the proposed approach, a feed-forward ANN is utilized to predict the channel status in order to improve the overall efficiency and throughput of spectrum bands. Furthermore, an improved TLBO (iTLBO) algorithm is proposed and used to find optimal weights and biases of the ANN in training process. The proposed iTLBO-ANN model is compared with some other prediction models in terms of prediction error and prediction efficiency [23].

\section{A. Teaching-Learning-Based Optimization (TLBO)}

Proposed by Rao et al. [15], TLBO algorithm is a simple and 
efficient population-based optimization technique which models the effect of a teacher on knowledge of the students in a classroom. A group of randomly generated learners (students) are considered as initial population. The knowledge of the students is evaluated by a specific fitness function. A person with the minimal objective function is chosen as teacher who shares his/her knowledge with other students. The quality of the students directly depends on the quality of the teacher knowledge. To increase the knowledge of the class, two different phases called "teacher phase" and "student phase" are implemented in TLBO process.

In teacher phase, the teacher tries to modify the knowledge of the students by shifting the mean value of them $\left(X_{\text {mean }}\right)$ toward a new mean $\left(X_{\text {teacher }}\right)$ as

$$
\begin{gathered}
X_{i, \text { new }}(k)=X_{i}(k)+\operatorname{rand}\left(X_{\text {teacher }}(k)\right. \\
\left.-T_{F} X_{\text {mean }}(k)\right)
\end{gathered}
$$

where $k$ is the iteration number and rand is a random number changing in the range of 0 to $1 . T_{F}$ is the teaching factor and randomly determined equal to 1 or 2 . A modified student $X_{i, n e w}$ replaces $X_{i}$ if it gives a better function value.

In the student phase, the students improve their knowledge (and so, the average fitness of the entire population) through interactions among themselves. Two students $X_{i}$ and $X_{j}(i \neq$ $j$ ) are randomly selected to interact based on the following mathematical expressions:

$$
\begin{aligned}
X_{i, \text { new }}(k)=X_{i}(k) & \\
& +\operatorname{rand}\left(X_{i}(k)\right. \\
& \left.-X_{j}(k)\right) ; \quad \text { if } f\left(X_{i}\right) \\
& >f\left(X_{j}\right) \\
X_{i, \text { new }}(k)=X_{i}(k) & \\
& +\operatorname{rand}\left(X_{j}(k)\right. \\
& \left.-X_{i}(k)\right) ; \\
& >f\left(X_{i}\right)
\end{aligned}
$$

where $f($.$) means the objective function of corresponding$ solution. Again, $X_{i, n e w}$ is accepted if it gives a better function value than $X_{i}$.

It can be seen that the teacher phase efforts to improve the convergence rate, while the student phase aims to enhance the population diversity.

\section{B. Improved TLBO}

Proposed In order to balance between exploitation and exploration ability of TLBO algorithm, a novel improved version of TLBO algorithm (iTLBO) is presented in three aspects to incorporate into original TLBO. These measures are carried out as follows:

\section{1) Opposition-Based initialization}

The population of the TLBO is initialized randomly. A more uniform distribution of the random guess in the solution space increases the convergence speed of the algorithm. On the one hand, for an efficient initial guess and hence a fast convergence, all directions of solution space should be looked simultaneously. On the other hand, without any a-priori knowledge, it is not possible to generate a uniform random population in the solution space. So, for the initial random population $\boldsymbol{X}:=\left[X_{1}, X_{2}, \ldots, X_{i}, \ldots, X_{n}\right]$, where $X_{i} \in\{a, b\}$ and $n$ is population size, an opposite population $\widetilde{\boldsymbol{X}}:=$ $\left[\tilde{X}_{1}, \tilde{X}_{2}, \ldots, \tilde{X}_{i}, \ldots, \tilde{X}_{n}\right], \tilde{X}_{i} \in\{a, b\}$ is defined [26], where

$$
\tilde{X}_{i}=a+b-X_{i} ; \quad i=1, \ldots, n
$$

The individual $\tilde{X}_{i}$ is selected for initial population if $f\left(\tilde{X}_{i}\right)>$ $f\left(X_{i}\right)$. However, a bi-directional search in the solution space increases the chance of approaching the global solution and also decreases the running time [26].

\section{2) Experienced teacher}

Unlike a real teaching-learning system in which the students may learn any proportion of the teacher's knowledge, the student can only learn nothing $\left(T_{F}=1\right)$ or all the things $\left(T_{F}=\right.$ 2 ) from the teacher in original TLBO [15-17]. In the proposed experience-oriented learning system, the experienced teacher teaches the worse learner more than the better learner. This can be model by an adaptive teaching factor in teacher phase. In each iteration, the adaptive $T_{F}$ for the student $X_{i}$ is modified as

$$
T_{F}=2-\frac{f\left(X_{i}\right)}{f\left(X_{\text {teacher }}\right)}
$$

The adaptive variation of the teaching factor during the search can be improve both the convergence speed and the exploration capability of the TLBO algorithm.

\section{3) Self learning}

In self-learning, the students can use their accumulated historical experience to learn in addition to the knowledge of others. Having a degree of blindness, the learning of the student is guided to their own gradient information [17, 27]. Considering self-learning, the student phase is implemented for iteration $\mathrm{k}$ as follow

$$
\begin{aligned}
X_{i, \text { new }}(k)=X_{i}(k) & +\operatorname{rand}\left(X_{i}(k)-X_{j}(k)\right) \\
& +\operatorname{rand}\left(X_{i}(k)-X_{i}(k\right. \\
& -1)) ; \quad \text { if } f\left(X_{i}\right)>f\left(X_{j}\right) \\
X_{i, \text { new }}(k)=X_{i}(k) & +\operatorname{rand}\left(X_{i}(k)-X_{j}(k)\right) \\
& +\operatorname{rand}\left(X_{i}(k)-X_{i}(k\right. \\
& -1)) ; \quad \text { if } f\left(X_{j}\right)>f\left(X_{i}\right)
\end{aligned}
$$

To verify the performance of the iTLBO algorithm, it applied to 10 benchmark functions. These functions are listed in Table1. Range denotes the searching space of the decision variables and $\boldsymbol{f}_{\text {min }}$ is the global optimum. To compare the performance of the 
TABLE I

BENCHMARK FUNCTIONS

\begin{tabular}{|c|c|c|c|}
\hline Function & Formula & Range & $f_{\min }$ \\
\hline$f 1$ (Sphere) & $f_{1}(x)=\sum_{i=1}^{D} x_{i}^{2}$ & {$[-100,100]$} & 0 \\
\hline f2 (Sum Square) & $f_{2}(x)=\sum_{i}^{D} i x_{i}^{2}$ & {$[-100,100]$} & $\mathbf{0}$ \\
\hline$f 3$ (Quadric) & $f_{3}(x)=\sum_{i=1}^{D}\left(\sum_{i=1}^{i} x_{j}^{2}\right)$ & {$[-100,100]$} & $\mathbf{0}$ \\
\hline$f 4($ Zakharov $)$ & $f_{4}(x)=\sum_{i=1}^{D} x_{i}^{2}+\left(\sum_{i=1}^{D} 0.5 i x_{i}\right)^{2}+\left(\sum_{i=1}^{D} 0.5 i x_{i}\right)^{4}$ & {$[-10,10]$} & $\mathbf{0}$ \\
\hline$f 5$ (Schwefel's P2.22) & $f_{5}(x)=\sum_{i=1}^{D}\left|x_{i}\right|+\prod_{i=1}^{D}\left|x_{i}\right|$ & {$[-10,10]$} & $\mathbf{0}$ \\
\hline$f 6$ (Rosenbrock) & $f_{6}(x)=\sum_{i=1}^{D-1}\left\{100\left(x_{i}^{2}-x_{i+1}\right)^{2}+\left(x_{i}-1\right)^{2}\right\}$ & {$[-30,30]$} & $\mathbf{0}$ \\
\hline f7 (Ackley) & $f_{7}(x)=\sum_{i=1}^{D}-20 \exp \left(-0.2 \sqrt{\frac{1}{D} \sum_{i=1}^{D} x_{i}^{2}}\right)$ & {$[-32,32]$} & 0 \\
\hline & $-\exp \left(\frac{1}{D} \sum_{i=1}^{D} \cos \left(2 \pi x_{i}\right)\right)+20+e$ & & \\
\hline$f 8$ (Rastrigin) & $f_{8}(x)=\sum_{i=1}^{D}\left(x_{i}^{2}-10 \cos \left(2 \pi x_{i}\right)+10\right)$ & {$[-5.12,5.12]$} & 0 \\
\hline$f 9($ Salmon $)$ & $f_{9}(x)=1-\cos \left(2 \pi \sqrt{\sum_{i=1}^{D} x_{i}^{2}}\right)+0.1 \sqrt{\sum_{i=1}^{D} x_{i}^{2}}$ & {$[-100,100]$} & 0 \\
\hline$f 10($ Griewank $)$ & $f_{10}(x)=\frac{1}{4000} \sum_{i=1}^{D} x_{i}^{2}-\prod_{i=1}^{D} \cos \left(\frac{x}{\sqrt{i}}\right)+1$ & {$[-600,600]$} & $\mathbf{0}$ \\
\hline
\end{tabular}

TABLE II

THE COMPARATIVE RESULTS OF ITLBO WITH OTHER OPTIMIZATION ALGORITHMS

\begin{tabular}{|c|c|c|c|c|c|c|c|}
\hline Function & & CLPSO & jDE & ABC & TLBO & IETLBO & iTLBO \\
\hline \multirow{2}{*}{$f 1$} & Mean & $1.65 \mathrm{E}-35$ & $1.12 \mathrm{E}-51$ & $1.43 \mathrm{E}-78$ & $1.00 \mathrm{E}-121$ & $0.00 E+00$ & $0.00 \mathrm{E}+00$ \\
\hline & Std. & $1.01 \mathrm{E}-35$ & $1.56 \mathrm{E}-51$ & $1.17 \mathrm{E}-78$ & $0.00 \mathrm{E}+00$ & $0.00 \mathrm{E}+00$ & $0.00 \mathrm{E}+00$ \\
\hline \multirow{2}{*}{$f 2$} & Mean & $7.03 E-20$ & $6.23 \mathrm{E}-18$ & $3.41 \mathrm{E}-25$ & $7.04 \mathrm{E}-43$ & $1.71 \mathrm{E}-87$ & $0.00 \mathrm{E}+00$ \\
\hline & Std. & $2.09 \mathrm{E}-20$ & $1.45 \mathrm{E}-18$ & $2.89 \mathrm{E}-25$ & $4.19 \mathrm{E}-43$ & $1.83 \mathrm{E}-88$ & $0.00 \mathrm{E}+00$ \\
\hline \multirow{2}{*}{$f 3$} & Mean & $2.60 \mathrm{E}-14$ & $1.73 \mathrm{E}-13$ & $2.43 \mathrm{E}-12$ & $1.13 \mathrm{E}-33$ & $2.03 E-76$ & $0.00 \mathrm{E}+00$ \\
\hline & Std. & $1.18 \mathrm{E}-14$ & $1.14 \mathrm{E}-13$ & $1.12 \mathrm{E}-12$ & $1.51 \mathrm{E}-33$ & $1.41 \mathrm{E}-76$ & $0.00 \mathrm{E}+00$ \\
\hline \multirow{2}{*}{$f 4$} & Mean & $5.71 \mathrm{E}-10$ & $2.11 \mathrm{E}-19$ & $4.19 E-45$ & $9.01 \mathrm{E}-88$ & $1.69 \mathrm{E}-110$ & $0.00 \mathrm{E}+00$ \\
\hline & Std. & $2.01 \mathrm{E}-10$ & $2.50 \mathrm{E}-19$ & $1.81 \mathrm{E}-45$ & $1.53 \mathrm{E}-88$ & $0.00 \mathrm{E}-00$ & $0.00 \mathrm{E}+00$ \\
\hline \multirow{2}{*}{$f 5$} & Mean & $5.71 \mathrm{E}-9$ & $3.19 \mathrm{E}-11$ & $1.73 \mathrm{E}-50$ & $1.35 \mathrm{E}-38$ & $5.01 \mathrm{E}-42$ & $4.19 \mathrm{E}-115$ \\
\hline & Std. & $3.55 \mathrm{E}-9$ & $1.43 \mathrm{E}-11$ & $1.03 \mathrm{E}-51$ & $1.51 \mathrm{E}-38$ & $4.00 \mathrm{E}-42$ & $1.35 \mathrm{E}-115$ \\
\hline \multirow{2}{*}{$f 6$} & Mean & $2.23 \mathrm{E}-11$ & $5.34 \mathrm{E}-25$ & $2.17 \mathrm{E}-20$ & $1.73 \mathrm{E}-61$ & $5.06 \mathrm{E}-86$ & $7.05 \mathrm{E}-108$ \\
\hline & Std. & $1.18 \mathrm{E}-11$ & $1.33 \mathrm{E}-26$ & $2.01 \mathrm{E}-20$ & $2.28 \mathrm{E}-62$ & $4.30 \mathrm{E}-86$ & $6.30 \mathrm{E}-108$ \\
\hline \multirow{2}{*}{$f 7$} & Mean & $8.14 \mathrm{E}-9$ & $3.45 \mathrm{E}-19$ & $1.83 \mathrm{E}-21$ & $7.36 \mathrm{E}-29$ & 5.11E-95 & $1.55 \mathrm{E}-92$ \\
\hline & Std. & $4.18 \mathrm{E}-10$ & $2.71 \mathrm{E}-19$ & $2.54 \mathrm{E}-21$ & $6.63 \mathrm{E}-29$ & $5.03 E-96$ & $8.01 \mathrm{E}-92$ \\
\hline \multirow{2}{*}{ f8 } & Mean & $3.11 \mathrm{E}-19$ & $6.18 \mathrm{E}-18$ & $3.24 \mathrm{E}-16$ & $2.19 \mathrm{E}-13$ & $1.41 \mathrm{E}-42$ & $1.12 \mathrm{E}-88$ \\
\hline & Std. & $2.21 \mathrm{E}-19$ & 1.17E-18 & $8.13 \mathrm{E}-15$ & $1.42 \mathrm{E}-13$ & $4.16 \mathrm{E}-42$ & $3.62 E-89$ \\
\hline \multirow{2}{*}{$f 9$} & Mean & $9.05 \mathrm{E}-22$ & $1.82 \mathrm{E}-21$ & $6.35 \mathrm{E}-52$ & $2.75 \mathrm{E}-80$ & $8.05 E-92$ & $5.15 \mathrm{E}-84$ \\
\hline & Std. & $1.67 \mathrm{E}-23$ & $1.90 \mathrm{E}-21$ & $1.56 \mathrm{E}-52$ & $1.60 \mathrm{E}-80$ & $7.20 \mathrm{E}-92$ & $0.00 \mathrm{E}+00$ \\
\hline \multirow[b]{2}{*}{$f 10$} & Mean & $4.12 \mathrm{E}-8$ & $1.55 \mathrm{E}-17$ & $1.84 \mathrm{E}-50$ & $5.60 \mathrm{E}-35$ & $1.17 \mathrm{E}-42$ & $4.45 \mathrm{E}-115$ \\
\hline & Std. & $2.60 \mathrm{E}-8$ & $2.45 \mathrm{E}-18$ & $2.50 \mathrm{E}-50$ & $4.32 \mathrm{E}-35$ & $8.65 E-43$ & $0.00 \mathrm{E}+00$ \\
\hline
\end{tabular}

proposed iTLBO, the experiments are also carried out on some other population-based optimization algorithms, including CLPSO [28], jDE [29], ABC [21], TLBO [15-17], and IETLBO [30]. Mean results of the 100 runs of the six algorithms on the 10 benchmark functions with 50 dimensions are summarized in Table 2, wherein the best results are shown in bold. In this table, 'Mean' is the mean result among 100 runs, and 'Std' is the standard deviation.

Table 2 shows that iTLBO is outperforms other algorithms in terms of mean solution and standard deviation for all benchmark functions except $\mathrm{f7}$ and mean solution of $\mathrm{f9}$. This indicates the ability of iTLBO algorithm to solve complex problems. For function $\mathrm{f} 7$, the mean best solution and standard deviation of IETLBO are smaller than those of other algorithms. Also for function $\mathrm{f} 9$, the mean solution of the IETLBO is the best.

\section{C. . iTLBO-ANN Predictor Scheme}

Although BP algorithm is the mostly used artificial neural network, there are some inherent defects in it. Firstly, it easily 


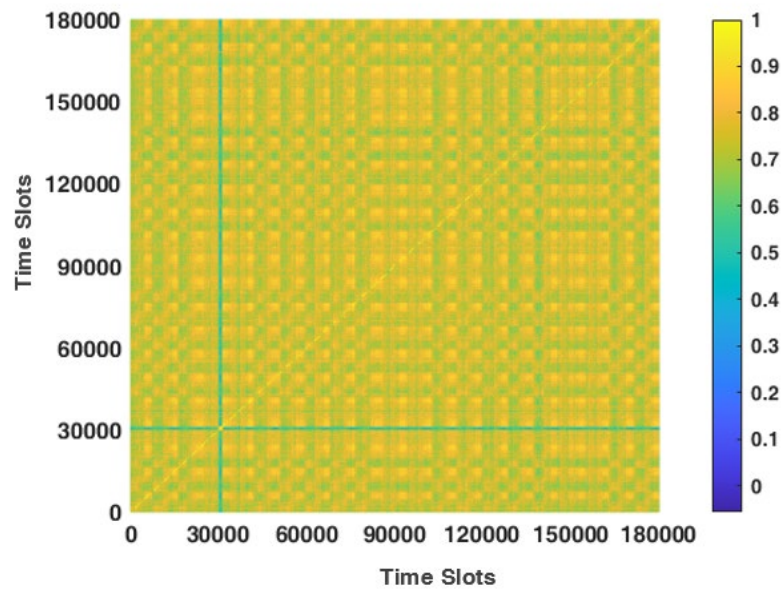

(a)

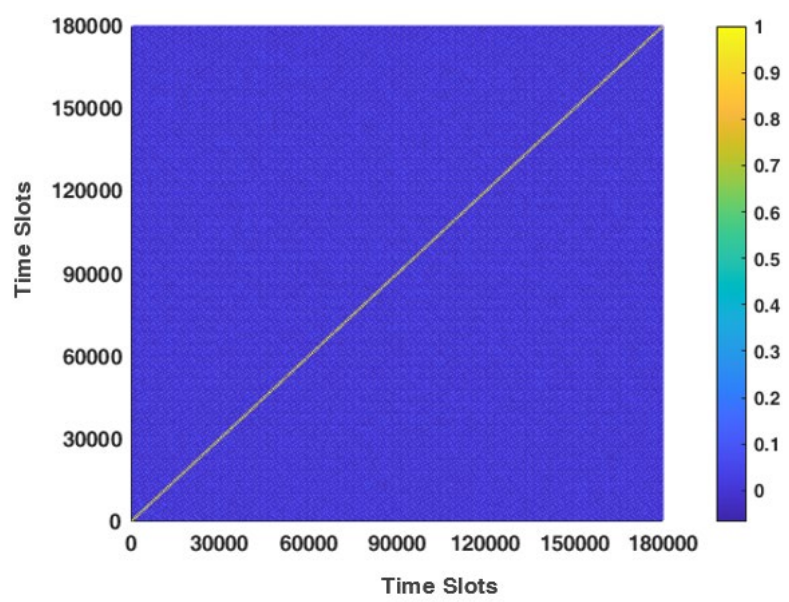

(b)

Fig. 2. Correlations in time domain

falls into local optimum. Then, for numerous uncertain CR channel data, the network cannot converge and so, the global optimal predictive model is hard to obtain by such a gradientbased algorithm. Second, it convergences very slowly. The speed of the BP-ANN convergence depends very much on the algorithm parameters such as learning rate and momentum factor as well as the size of derivative of related activation function [24-25, 31]. On the other hand, fast convergence speed and efficient global optimum searching ability are two important advantages of iTLBO algorithm. So, a hybrid iTLBO-ANN scheme is used to simultaneously take advantages of both ANN and iTLBO algorithm for spectrum prediction in cognitive radios. In this scheme, ANN is used to model the traffic characteristic of spectrum channel and iTLBO algorithm is used for ANN training to increase the accuracy and efficiency of ANN-based channel status predictor. In fact, the iTLBO is applied to select the optimal weights and threshold values of ANN during training process.

In this regard, a population of solutions is randomly initialized, in which each solution consists of the ANN parameters (weights and threshold values) to be updated. Then, the population is evolved through the knowledge improvement of students to obtain optimal solution with minimum error rate. In this case, a sample of training data, which is related to channel status data, is applied as the input variable to ANN. The optimal parameter search process is evaluated using mean square error function as the fitness function. These error criteria are applied to calculate the objective function value of each solution (student) in iTLBO algorithm. The best solution, who gives the minimum value of the fitness function, is driven to the next generation as teacher. Weights and threshold values are consistently updated until stopping criterion is satisfied.

\section{EXPERIMENTAL EVALUATION}

Use The real-world spectrum data, measured for 925$960 \mathrm{MHz}$ frequency band, is used to demonstrate the effectiveness of the proposed iTLBO-ANN scheme. The data have been prepared during approximately four months using a radiometer. The resolution bandwidth of individual spectrum band is $0.2 \mathrm{MHz}$ ( 175 frequency bins) and the inter-sample time is $1 \mathrm{~min}$, which results in 180000 samples for 125 days from September 23, 2018 to January 18, 2019. The value of NF is measured as $-40 \mathrm{dbm}$.

To analyze the temporal correlations of the real-world spectrum dataset, the Pearson correlation coefficient (PCC) method [32] is used. The PCC between two vector random variables $\boldsymbol{a}$ and $\boldsymbol{b}$ is defined as

$$
\rho_{a, b}=\frac{\operatorname{cov}(\boldsymbol{a}, \boldsymbol{b})}{\sigma_{\boldsymbol{a}} \sigma_{\boldsymbol{b}}}=\frac{E\left[\left(\boldsymbol{a}-\mu_{\boldsymbol{a}}\right)\left(\boldsymbol{b}-\mu_{\boldsymbol{b}}\right)\right]}{\sigma_{\boldsymbol{a}} \sigma_{\boldsymbol{b}}}
$$

where $\mu_{\boldsymbol{a}}$ and $\mu_{\boldsymbol{b}}$ are the true means and $\sigma_{\boldsymbol{a}}$ and $\sigma_{\boldsymbol{b}}$ are the true standard deviations of vector random variables $\boldsymbol{a}$ and $\boldsymbol{b}$, respectively. The value of $\rho_{\boldsymbol{a}, \boldsymbol{b}}$ ranges from +1 to -1 . A value of +1 (or -1 ) indicates that $\boldsymbol{a}$ is fully positively (or negatively) correlated to $\boldsymbol{b}$ and a value of 0 shows that $\boldsymbol{a}$ is not correlated to $\boldsymbol{b}$ at all. Figure 2a represents the correlations in time domain for the real-world spectrum data matrix $\mathbf{X}$. To do this, $\boldsymbol{a}$ and $\boldsymbol{b}$ variables in Equation 22 are replaced by two columns $x_{, i}, i \in$ $\{1, \ldots, T\}$ and $x_{, j}, j \in\{1, \ldots, T\}$, respectively. Compared to the Gaussian noise data with close to zero correlation matrix (Fig. $2 \mathrm{~b}$ ), the real-world data display significant time correlations (Fig. 2a). As can be seen, there are high correlations between adjacent and far apart time slots. In should be noted that in some of the time slots, most of the frequency bands are idle. So, just like a noisy signal, the correlations are low. This reason justifies the low correlation regions in Fig. 2a.

\section{A. Evaluation Metrics}

Although The spectrum prediction performance is quantified in terms of prediction error and prediction efficiency metrics. Mean absolute error (MAE) and root mean square error (RMSE) are used to evaluate the performance of the ANN in combination with evolutionary algorithm as follows: 
TABLE III

The parameters of TLBO, GA and FF algorithms

\begin{tabular}{ll|lc|lc}
\hline \multicolumn{1}{c|}{ TLBO Parameters } & \multicolumn{2}{c}{ GA Parameters } & \multicolumn{2}{c}{ FF Parameters } \\
\hline Population size & 60 & Population size & 60 & Population size & 60 \\
Number of iterations & 500 & Crossover rate & 0.9 & Initial attractiveness & 1 \\
& & Mutation rate & 0.01 & Absorption coefficient & 1 \\
& & Number of iterations & 500 & Number of iterations & 500 \\
\hline
\end{tabular}

TABLE IV

The average and best values of MAE and RMSE

\begin{tabular}{|c|c|c|c|}
\hline Model & Index & Average\% & Best \% \\
\hline \multirow{2}{*}{ GA-ANN } & MAE & 7.20 & 6.55 \\
\cline { 2 - 4 } & RMSE & 7.80 & 7.36 \\
\hline \multirow{2}{*}{ FF-ANN } & MAE & 6.90 & 6.15 \\
\cline { 2 - 4 } & RMSE & 7.85 & 7.20 \\
\hline \multirow{2}{*}{ TLBO-ANN } & MAE & 6.95 & 6.10 \\
\cline { 2 - 4 } & RMSE & 7.70 & 6.94 \\
\hline \multirow{2}{*}{ iTLBO-ANN } & MAE & $\mathbf{3 . 0 5}$ & $\mathbf{2 . 6 0}$ \\
\cline { 2 - 4 } & RMSE & $\mathbf{4 . 5 5}$ & $\mathbf{4 . 2 5}$ \\
\hline
\end{tabular}

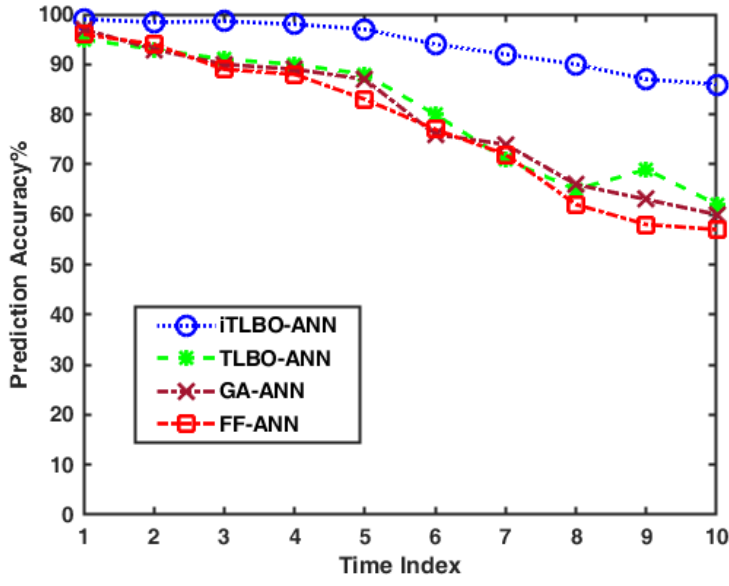

Fig. 3. Prediction Accuracy (PA) of the prediction models

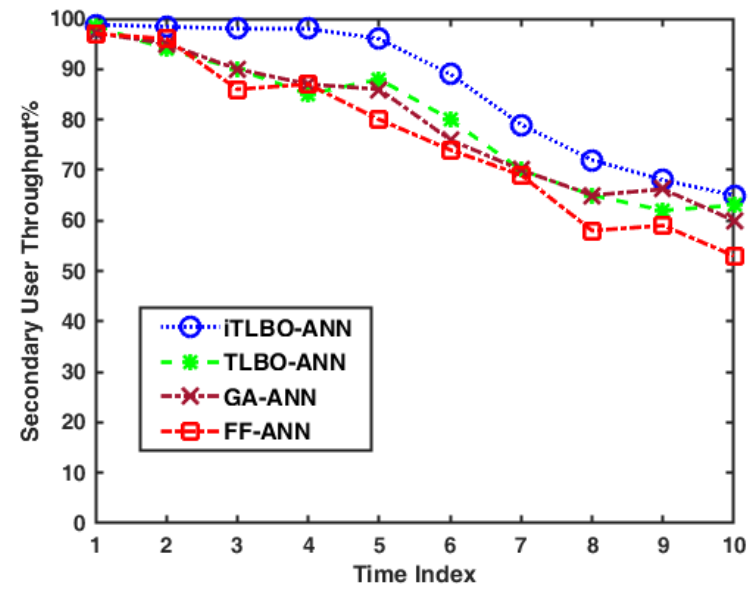

Fig. 4. Secondary User Throughput (SUT) of the prediction models

$$
\begin{aligned}
M A E & =\frac{1}{n} \sum_{i=1}^{n}\left|y_{i, \text { Actual }}-y_{i, \text { Predicted }}\right| \\
R M S E & =\left[\frac{1}{n} \sum_{i=1}^{n}\left(y_{i, \text { Actual }}-y_{i, \text { Predicted }}\right)^{2}\right]^{1 / 2}
\end{aligned}
$$

where $y_{i, \text { Actual }}$ and $y_{i, \text { Predicted }}$ are the actual and predicted spectrum data, respectively. The smaller the RMSE and MRE, the more accurate and stable the predicted output. To evaluate the prediction efficiency, two well-used metrics including prediction accuracy (PA) and secondary user throughput (SUT) are applied for each time-slot. For each time-slot $d_{., j}$, the PA is defined as

$$
P A=\frac{N_{P-\text { correct }}}{N}
$$

where $N$ is the total number of frequency channels and $N_{P-\text { correct }}$ is the number of frequency channels that are correctly predicted. For each time-slot, the SUT as a measure of secondary user spectrum utilization is calculated by

$$
S U T=\frac{N_{P-i d e l}}{N_{A-i d e l}}
$$

where $N_{P \text {-idel }}$ and $N_{A-i d e l}$ represent the number of frequency channel predicted to be idle and the number of actual idle frequency channels, respectively.

\section{B. Performance Comparison}

This sub-section evaluates the performance of the proposed iTLBO-ANN based channel prediction scheme in comparison with GA-ANN, FF-ANN and TLBO-ANN prediction models through simulation study. Like the iTLBO-ANN model in which the ANN is trained using iTLBO algorithm, GA, FF and TLBO are used to train the ANN in GA-ANN, FF-ANN and TLBO-ANN models, respectively. Table 3 shows the parameters and their settings for the TLBO, GA and FF algorithms, which were determined after some preliminary experiments. 
In simulations, a sliding window is applied to pick $T-1$ successive samples from spectrum data as the ANN inputs. The Tth sample is set as the desired output. The error threshold and the maximum number of iterations are set to $10^{-5}$ and $10^{3}$, respectively.

Table 4 provides the average and best values of MAE and RMSE. The prediction tests are carried out for 100 times independently. The number of hidden nodes are set to 15 . It is found from the table that iTLBO-ANN achieve the best performances in this case study. The average and best MAE values for the iTLBO-ANN are $3.05 \%$ and $2.60 \%$, respectively, which are lower than those of the other ANN-based prediction schemes. Thus, the average prediction of the iTLBO-ANN model is nearly $97 \%$. Furthermore, the smallest average and best RMSA values are obtained from the iTLBO-ANN models, i.e., $4.55 \%$ and $4.25 \%$. So, the iTLBO-ANN could maintain higher stability when predicting the channel status.

For assessing the prediction efficiency, a new scenario is designed in which 10 upcoming samples are predicted without updating the model. Fig. 3 and 4 clarify that the proposed iTLBO-ANN model has the best performances in terms of the PA and SUT metrics. The number of hidden nodes are set to 15 and the mean result of the 100 independent runs are reported. For the first five predictions, the iTLBO-ANN model provide quiet accurate results. However, since the model is not updated, the prediction success is degraded from beginning the sixth time-slot. It should be noted that despite the lower accuracy of other prediction models compared to the iTLBO-ANN model, the performance of these models in predicting the first five samples is also acceptable.

\section{CONCLUSION AND FUTURE WORK}

The A novel spectrum prediction scheme is presented to increase the spectrum efficiency of cognitive radio networks. In this scheme, a multi-layer ANN is used to model the traffic characteristic of spectrum channel and a new improved version of TLBO (iTLBO) algorithm is used for ANN training. The real-world spectrum data, measured for $925-960 \mathrm{MHz}$ frequency, is used to demonstrate the effectiveness of the proposed iTLBO-ANN prediction model. Performance evaluation via real-world spectrum dataset confirms the effectiveness and efficiency of the proposed model. The presence of measurement errors and missing data in historical observations and their effects on the proposed model will be investigated in the future work.

\section{REFERENCES}

[1] Jin, Z., Yao, K., Lee, B., Cho, J., Zhang, L. (2019). Channel Status Learning for Cooperative Spectrum Sensing in Energy-Restricted Cognitive Radio Networks. IEEE Access. 7, pp. 64946-64954.

[2] Mitola, J., Maguire, J.Q. (1999). Cognitive radio: making software radios more personal. IEEE Pers Commun. 6(4), pp.13-18.

[3] Bhowmick, A., Yadav, K., Roy, S.D., Kundu, S. (2017) Throughput of an energy harvesting cognitive radio network based on prediction of primary user. IEEE Trans Veh Technol. 66(9), pp.8119-8128.

[4] Xing, X., Jing, T., Cheng, W., Huo, Y., Cheng, X. (2013). Spectrum prediction in cognitive radio networks. IEEE Wireless Commun.20(2), pp. 90-96.
[5] Yarkan, S., Arslan, H. (2007). Binary Time Series Approach to Spectrum Prediction for Cognitive Radio. In: 66th IEEE Vehicular Technology Conference. Baltimore, MD, pp. 1563-1567.

[6] Tumuluru, V.K., Wang, P., Niyato, D. (2012). Channel status prediction for cognitive radio networks. Wireless Communications and Mobile Computing. 12(10), pp. 862-874.

[7] Yang, J., Zhao, H. (2015). Enhanced Throughput of Cognitive Radio Networks by Imperfect Spectrum Prediction. IEEE Commun Lett. 19(10), pp.1738-1741.

[8] Jing, T., Xing, X., Cheng, W., Huo, Y., Znati, T. (2013). Cooperative spectrum prediction in multi-PU multi-SU cognitive radio networks. In: 8th International Conference on Cognitive Radio Oriented Wireless Networks. Washington, DC, pp. 25-30.

[9] Shaghluf, N., Gulliver, T.A. (2018). Spectrum and energy efficiency of cooperative spectrum prediction in cognitive radio networks. Wireless Networks. 25(2), pp. 3265-3274.

[10] Chang, J., Huang, X. (2019). Spectrum Entropy Prediction Assisted Channel Selection for Secondary Users. IEEE Network. 33(3), pp.190195.

[11] Ding, G., et al. (2017). Robust Online Spectrum Prediction with Incomplete and Corrupted Historical Observations. IEEE Trans Veh Technol. 66(9), pp. 8022-8036.

[12] Omer, A.E. (2015). Review of spectrum sensing techniques in Cognitive Radio networks. In: 2015 International Conference on Computing, Control, Networking, Electronics and Embedded Systems Engineering (ICCNEEE). Khartoum, pp. 439-446.

[13] Eltabie, O.M., Abdelkader, M.F., Ghuniem, A.M. (2019). Incorporating Primary Occupancy Patterns in Compressive Spectrum Sensing. IEEE Access. 7, pp. 29096-29106.

[14] Hamdaoui, B., Khalfi, B., Guizani, M. (2018). Compressed Wideband Spectrum Sensing: Concept, Challenges, and Enablers. IEEE Communications Magazine. 56(4), pp. 136-141.

[15] Rao, R.V., Savsani, V.J., Vakharia, D.P. (2011). Teaching-learning-based optimization: a novel method for constrained mechanical design optimization problems. Comput Aided Des. 43(3), pp. 303-315

[16] Rao, R.V., Patel, V. (2013). An improved teaching-learning-based optimization algorithm for solving unconstrained optimization problems. Sci Iran. 20, pp. 710-720.

[17] Chen, X., Yu, K., Du, W., Zhao, W., Liu, G. (2016). Parameters identification of solar cell models using generalized oppositional teaching learning based optimization. Energy. 99, pp. 170-180

[18] Holland, J.H. (1975). Adaptation in Natural and Artificial Systems. University of Michigan Press, Ann Arbor, USA.

[19] Kennedy, J., Eberhart, R.C. (1995). Particle swarm optimization. In: IEEE Int. Conf. on Neural Networks, Washington DC, USA, pp. 1942-1948.

[20] Price, K., Storn, R., Lampinen, A. (2005). Differential Evolution-A Practical Approach to Global Optimization. Springer-Verlag, Berlin, Germany.

[21] Karaboga, D., Basturk, B. (2007). A powerful and efficient algorithm for numerical function optimization: Artificial Bee Colony (ABC) algorithm. J. Global Optim. 39(3), pp.459-471.

[22] Yang, X.S. (2009). Firefly algorithms for multimodal optimization. In: Watanabe O, Zeugmann T. (ed), Fifth Symposium on Stochastic Algorithms, Foundation and Applications, LNCS, pp. 169-178.

[23] Eltholth, A.A. (2016). Spectrum prediction in cognitive radio systems using a wavelet neural network. In: 24th International Conference on Software, Telecommunications and Computer Networks (SoftCOM), Split, pp. 1-6.

[24] Schalkoff, R.J. (2009). Artificial Neural Networks. John Wiley \& Sons, Ltd.

[25] Gudise, V.G., Venayagamoorthy, G.K. (2003). Comparison of particle swarm optimization and backpropagation as training algorithms for neural networks. In: IEEE Swarm Intelligence Symposium, IN, USA, pp. 110117.

[26] Tizhoosh, H.R. (2005). Opposition-Based Learning: A New Scheme for Machine Intelligence. In: International Conference on Computational Intelligence for Modelling, Control and Automation and International Conference on Intelligent Agents, Web Technologies and Internet Commerce, Vienna, pp. 695-701.

[27] Xiaoyuan, J., Hu, Y., Jianxin, Z., Yajun, Y., Xu, S. (2017). An improved teaching-learning-based optimization algorithm and its application to a combinatorial optimization problem in foundry industry. Appl Soft Comput. 57, pp. 504-516. 
[28] Liang, J.J., Qin, A.K., Suganthan, P.N., Baskar, S. (2006). Comprehensive learning particle swarm optimizer for global optimization of multimodal functions. IEEE Trans Evol Comput. 10(3), pp. 281-295

[29] Brest, J., Greiner, S., Boskovic, B., Mernik, M., Zumer, V. (2006). Selfadapting control parameters in differential evolution: a comparative study on numerical benchmark problems. IEEE Trans Evol Comput. 10(6), pp. 646-657.

[30] Zou, F., Wang, L., Hei, X., Chen, D. (2015). Teaching-learning-based optimization with learning experience of other learners and its application. Appl Soft Comput. 37, pp. 725-736.

[31] Benali, A., Hachama, M., Bounif, A. et al. (2021). A TLBO-optimized artificial neural network for modeling axial capacity of pile foundations. Eng Comput. 37, pp. 675-684.

[32] Pearson, K. (1895). Note on Regression and Inheritance in the Case of Two Parents. In: Proceedings of the Royal Society of London, pp. 240242. 